\title{
Using Physlets to teach electrostatics
}

\author{
Scott W. Bonham and John S. Risley \\ Department of Physics, North Carolina State University, Raleigh, NC 27695 \\ Wolfgang Christian \\ Department of Physics, Davidson College, Davidson, NC 28036 \\ Published in The Physics Teacher, $v 57$ (1999) pp. 276-281
}

\section{Introduction}

One of the great difficulties in teaching electrostatics is the abstract nature of the subject. Fields are difficult to visualize, the mathematical relationships can be complex, and few students enter the course with personal experience of the subject. Simulations such as EM Field ${ }^{1}$ and Electric Field Hockey $^{2}$ can provide valuable help to students in visualizing and interacting with electrostatic phenomena. However, ensuring that students work on them effectively either inside or outside of class can be a daunting task for an instructor, as well as the logistics of outfitting a computer laboratory with these simulations. A solution to this problem is to use the World Wide Web and Java applets. ${ }^{3}$ Students can use any computer with an Internet connection, and student responses can be directly collected through the Web to monitor compliance. In this article we describe six exercises in electrostatics, how they were used at NCSU, and give suggestions how they might be used in other contexts. These type of problems work well with a web-based assessment system such as NCSU's WebAssign, ${ }^{4}$ which both delivers and evaluates student work. ${ }^{5}$ However, such a system is not a requirement for using Physlet problems. These exercises are available both in a WebAssign version and a straight html version; information on obtaining them is at the end of the article.

These six exercises cover some of the most important topics in electrostatics and demonstrate how this technology can be applied to physics instruction. The topics covered are: the operational definition of charge, the $1 / \mathrm{r}^{2}$ nature of Coulomb's Law, superposition, representations of electric fields and potentials, the concept of electric flux, and the interplay of electric potential and energy conservation. These exercises were used as in-class exercises in a computer classroom (part of the SCALE-UP_-Student-Centered Activities for Large Enrollment University Physics_curriculum project $^{6}$ ). One networked, notebook computer was available to each group of three students. With suitable guidance, most could be used as homework exercises. These exercises use the EField Physlet from Davidson College. 7

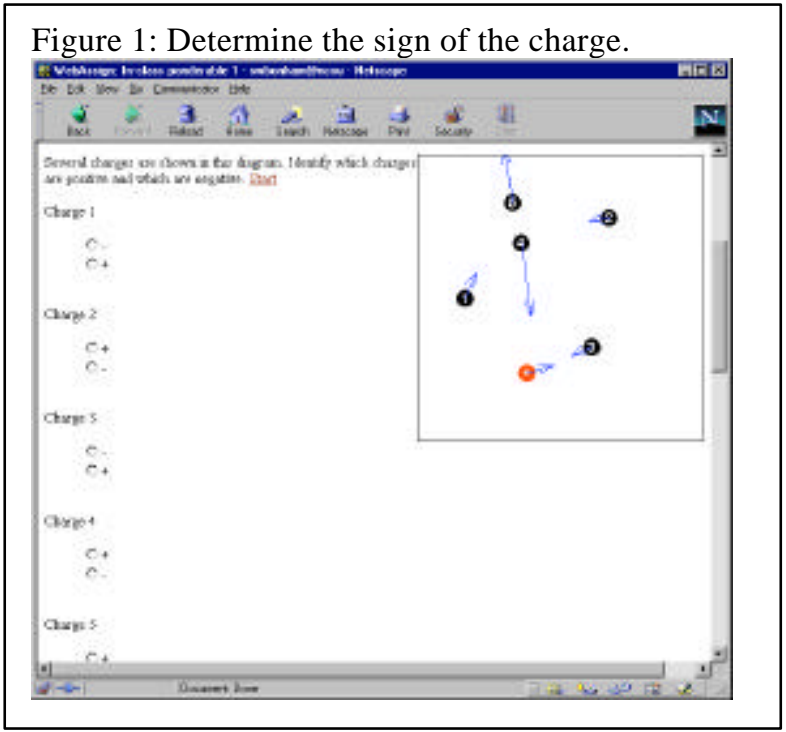

Determine the sign of the charge

The first exercise serves both to reinforce the operational definition of charge ("opposites attract, likes repel") and to introduce students to using Physlet problems. When the exercise is loaded, you see the layout shown in Figure 1. There is a box with five numbered black charges and a red charge marked with a "+". A blue arrow represents the total force acting on each charge. As you move the charges, the forces change appropriately. The accompanying question asks you to determine the sign of each charge, and to select the corresponding radio buttons. This task is more complex than it first seems. You are basically comparing the force on each charge with the marked reference charge. One strategy is to put one charge in each of the four corners of the screen and one in the middle next to the test charge, and then systematically exchange the charge in the middle with the rest of the charges. Other strategies can be deployed. This exercise allows you to show some creativity in solving a physics problem.

In our class this exercise followed a short introduction to the nature of charge, including the two types of charges. Students were instructed to go to their computers, log in to WebAssign, and work in groups on this exercise. This exercise proved to be easy for most students once they realized that the charges could be moved. Initially, many students 
thought the Physlet was just a static image.

Following the exercise, groups wrote their answers on the board to share with the class. This exercise should work well as a homework exercise as long as the instructor has given a brief demonstration of how to use the Physlet.

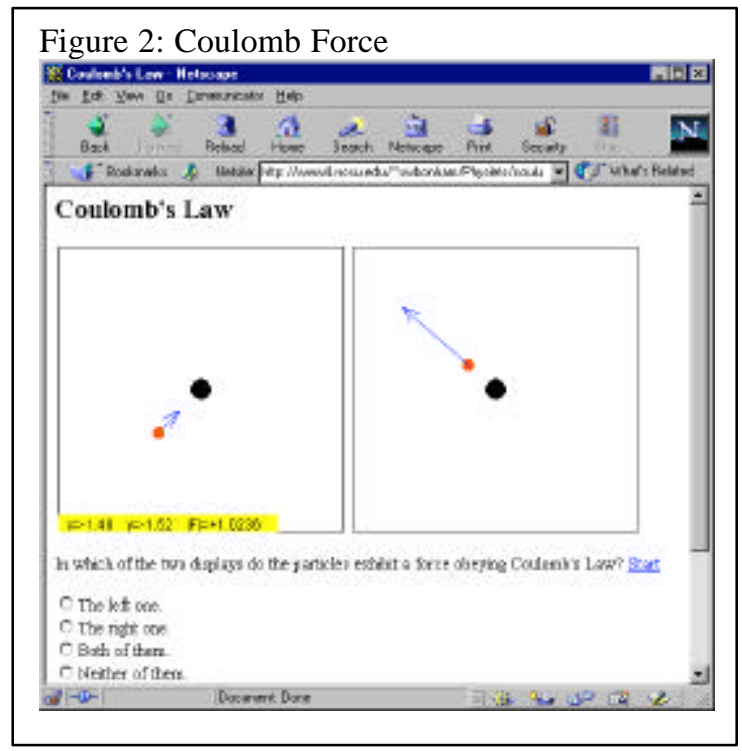

\section{Coulomb force}

The next exercise addresses a fundamental relationship of charges-Coulomb's law. In this exercise, you see two boxes with a solid black charge inside each, as shown in Figure 2. There is a small, movable test charge in each box with an arrow that shows the field strength and direction of the electric field. When you click on the test charge, a yellow box appears in the lower left-hand corner of the box displaying the position of the test charge and the force acting on it. The question is "In which of the two displays do the particles exhibit a force obeying Coulomb's law?" The multiple choice answers are the right box, the left one, both or neither. By moving the test charge, you can find the magnitude of the force on the test charge at various locations.

This exercise was used in our classroom during the period following the first exercise. Again, the students were asked to do the exercise in groups. Initially, many students concluded that both charges obeyed Coulomb's law since the force decreased with distance. Some groups then realized that the dependency of the force on distance was not the same in both cases and started to make measurements. Other groups were guided by the instructors to recognize that Coulomb's law has a specific relationship between distance and force, that is $\mathrm{F} \sim$ $1 / r^{2}$. These groups were easy to identify as they thought they had finished the task in less than five minutes. Most groups solved this problem successfully by first measuring the force at one position, and then at a location that was twice the distance from the center. In the left box, this procedure results in a force half the initial one; in the right box the second force is a fourth of the initial one, so it alone obeys Coulomb's Law. Afterwards, the instructor asked the groups to share their results and the different strategies that they employed. This exercise took a total of 20 and 30 minutes. This exercise could be used as homework problem if students were explicitly told they needed to measure the force at several positions to determine whether Coulomb's law is obeyed. However, the initial guidance would deny your students the learning opportunity of figuring out for themselves that Coulomb's law is not just any force decreasing with distance and that they need to check this dependence quantitatively.

\section{Unknown charge}

The principle of superposition is an important part of electrostatics with which many students need practice. This exercise provides practice using the simplest case-two point charges. In this exercise, you see a box with two solid black charges. A small, movable test charge is marked with an arrow displaying the direction and magnitude of the force acting on it. The position of the test charge and the magnitude of the force on it are displayed in the lower left-hand corner of the box when you click on the charge. You are told the amount of charge on one of the point charges and are asked to determine the magnitude on the other one. One way to solve this problem is to place the test charge near the known point charge to obtain the magnitude of the force at that point. Then you move the test charge to the same distance from the second point charge. The ratio of the forces is the ratio of the charges. A second method is to measure the force at two different positions, set this equal to the sum of the Coulombic forces from the two point charges, and

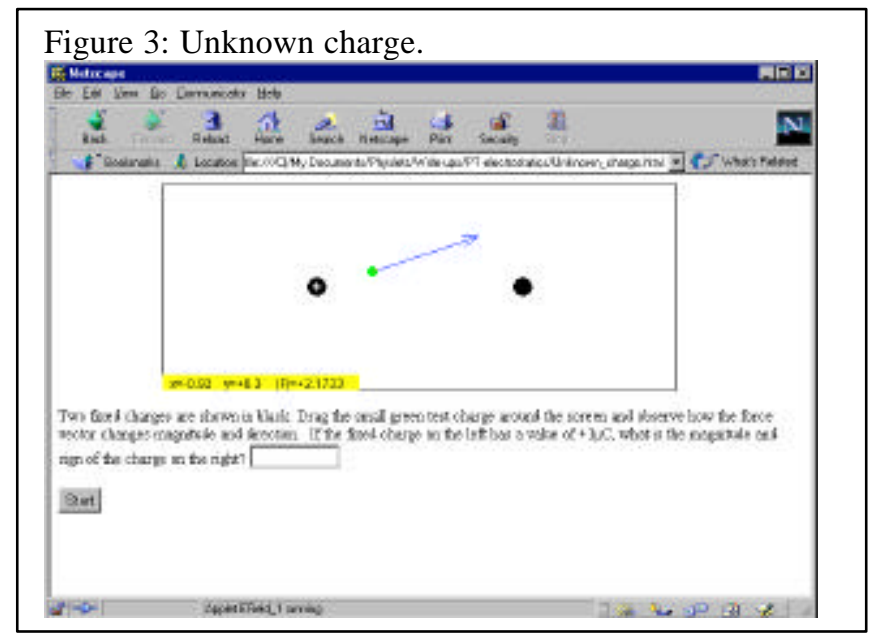


solve for the unknown charge.

This was a difficult exercise for the students and is more subtle than it first appears. The first method requires ratio reasoning, which is weak or lacking in many students. 8 The second method involves vector addition, another area of student difficulty, ${ }^{9}$ which is further complicated for students who are only familiar with SI units because EField uses simplified (or cgs) units where $F=q q / r^{2}$. In our classroom the students needed explicit guidance on how to use the ratio method in order to solve this exercise. Afterwards, each group shared their answer on the board. The variation among the results led to a good discussion on measurement and uncertainty. To assign this problem as a homework exercise, most students would probably need to be instructed to use ratio reasoning through an in-class example.

\section{Electric field representations}

The ability to interpret different representations of an abstract concept such as an electric fields is an important skill, but one with which students have difficulty. The next exercise helps address that problem. In it, you see two boxes. One box contains equipotential lines representing an electric field and the other is a vector field representation. The vector field representation is slightly different from the vector representation typically used at the introductory level, where magnitude is indicated by length. It is inherently difficult to set up arbitrary vectors with a computer; automatic computerized displays usually place the vectors on a regular grid, instead of strategic positions chosen for clarity by a textbook artist. In this situation, vectors longer than the grid spacing often produce confusing diagrams, while ones much shorter than the grid distance can not be easily seen. EField solves this difficulty using color for the magnitude of the vector instead of the length. We believe that the vector field representation did not produce significant difficulties for any students. The vector representation box contains one of four different vector fields. These vector fields are said to be the attempts of four hypothetical students to represent the equipotential line field and are displayed when the "student's" name is clicked. Students are asked which of these is the correct representation. Students can also measure the potential with the cursor to double-check themselves. This exercise is shown in Figure 4.

This exercise was used as a group exercise in our classroom. Most students had no difficulty with it. The important aspects for identifying the answer are the number of charge concentrations and

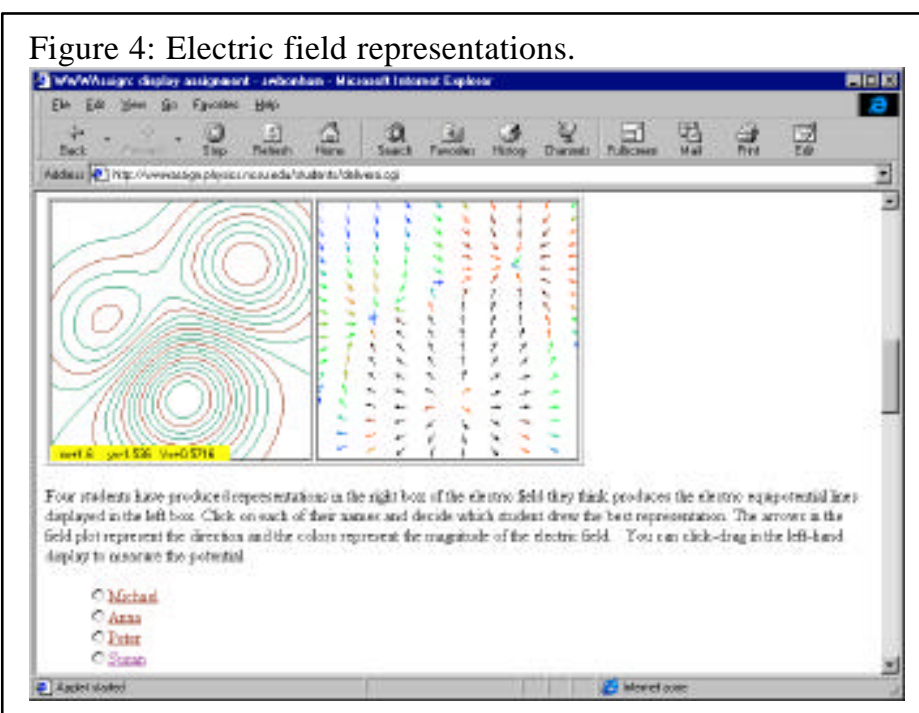

their relative signs. This question should work well as a homework exercise for students. The correct answer is the vector diagram shown in this figure.

\section{Electric flux through a cube}

The concepts of electric flux, closed surfaces, and Gauss's law provide multiple difficulties for students. The next exercise provides an opportunity for students to determine the flux through a cube either empty or containing a charged object. In this exercise, you see a box with a test charge inside, as in Figure 5. You can move the test charge to observe the direction and magnitude of the electric force vector at all points. You can also click the cursor anywhere and read the position and the magnitude of the electric field at that point. There are two different options to be explored, one has a uniform field and no charge inside the cube and the

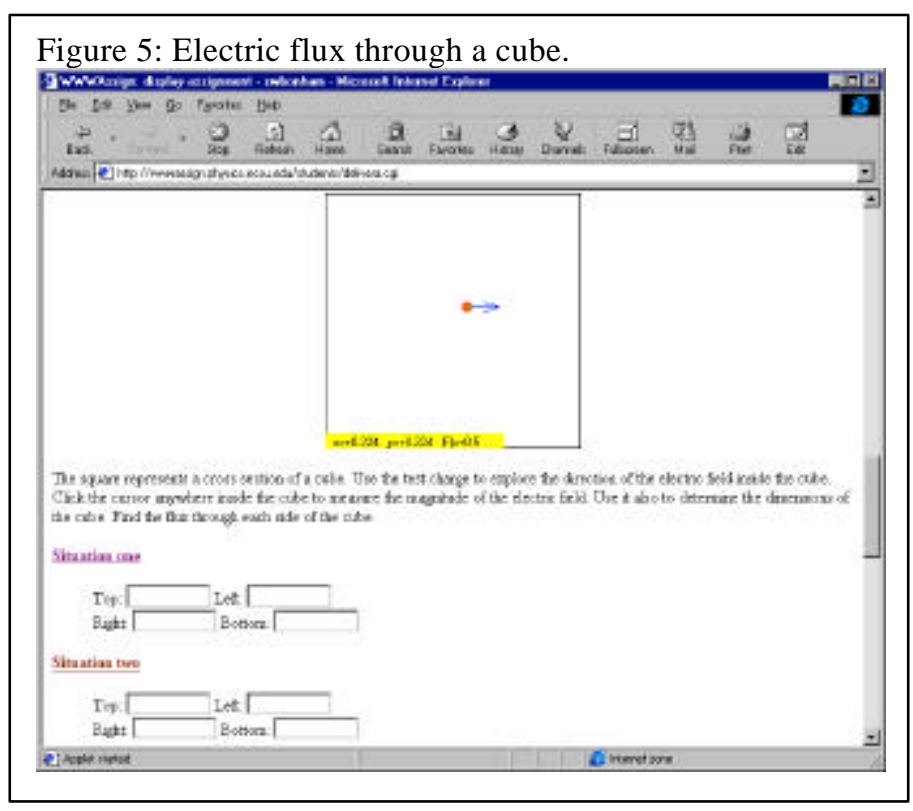


other has a uniform field but the direction switches in the middle of the box, corresponding to a sheet of charge enclosed by the cube. You are asked to determine the flux through the sides of the cube.

This exercise was used in our class following a review on the definition of electric flux. Students worked in groups, and no groups had any difficulty correctly applying the definition of flux to obtain positive, negative or zero values for the different sides. Some groups had difficulty visualizing how the twodimensional image was representing a threedimensional object. They needed help realizing that each of the four lines represented a side of the cube that extended out of the screen the same distance as the length of the line. Some students also asked the instructors for the length of each side, not realizing that they could get that information by clicking the cursor at various locations in the box. This exercise took less than ten minutes. If this exercise were to be used as a homework assignment, it would be important to explain the function of the test charge and cursor, and explain how the two-dimensional figure is representing a three-dimensional object.

\section{Particle in an uniform electric field}

This last exercise gave students the most difficulty. It is ties together several concepts from electrostatics and from mechanics. In this exercise you see a box with a test charge and a region of constant electric field in the middle. When you click "Play," the particle moves with an initial velocity toward the left. As the charge enters the field, it slows down at a constant rate. Clicking the cursor gives the coordinates and the magnitude of the electric field at that point. You are asked to determine the mass of the particle, given its charge. This exercise is shown in Figure 6.

This exercise is a complex, multi-step problem that most students needed some help with. First, they must measure the initial and final velocities of the particle by measuring the displacement for a given duration. Then they must use electrostatic relationships and either apply Newton's second lawthe relationships $\mathrm{a}=\Delta \mathrm{v} / \Delta \mathrm{t}, \mathrm{F}=$ ma and $\mathrm{F}=\mathrm{qE}$-or use conservation of energy from electrostatics, applying $\Delta \mathrm{U}=\mathrm{q} \Delta \mathrm{V}$, $\mathrm{K}=1 / 2 \mathrm{mv}{ }^{2}$ and $\Delta \mathrm{U}+\Delta \mathrm{K}=0$. Many students "forgot" the mechanics they were taught the previous semester. In addition,

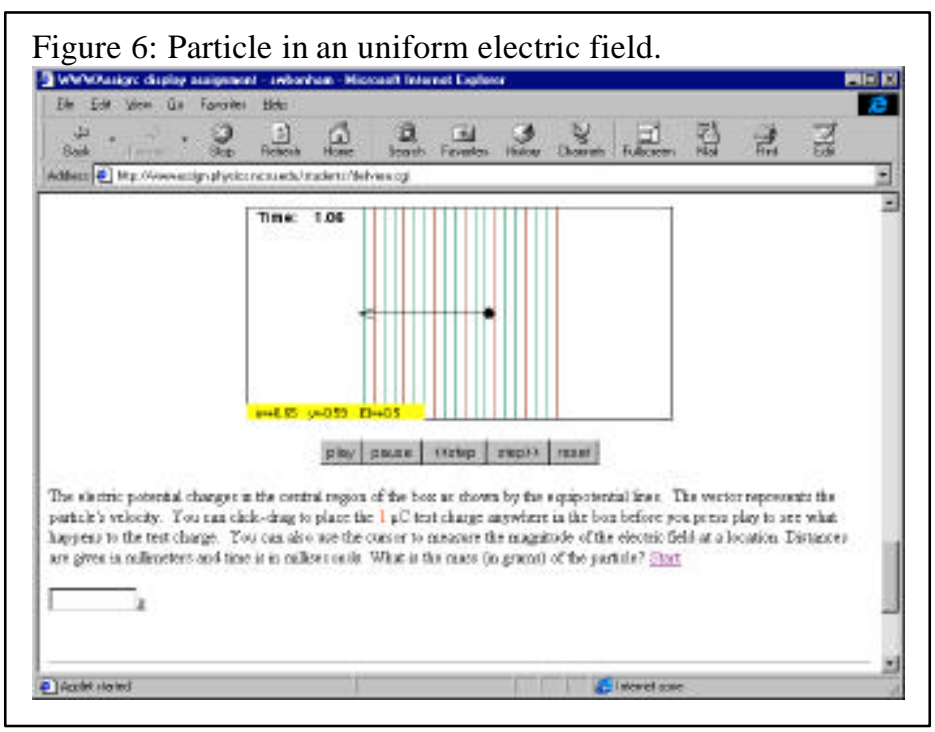

they had difficulty applying several principles at once. In our class, the exercise was assigned at the same time as the field representation problem. Although it required a half of an hour to work and help from the circulating instructors, it is a good comprehensive problem for tying together several different concepts in physics. To assign this problem as a homework exercise, most students would probably need to be instructed to use energy conservation or Newton's laws with an in-class example. In the WebAssign version of this problem, the charge on the particle is randomized so each student or group of students has a different value.

\section{Information for using these exercises}

Table 1: Example of the JavaScript for controlling EField. This example defines and places the charges in the exercise Determine the sign of the charge. Comments to the right of the double slash explain the action of the respective JavaScript methods.

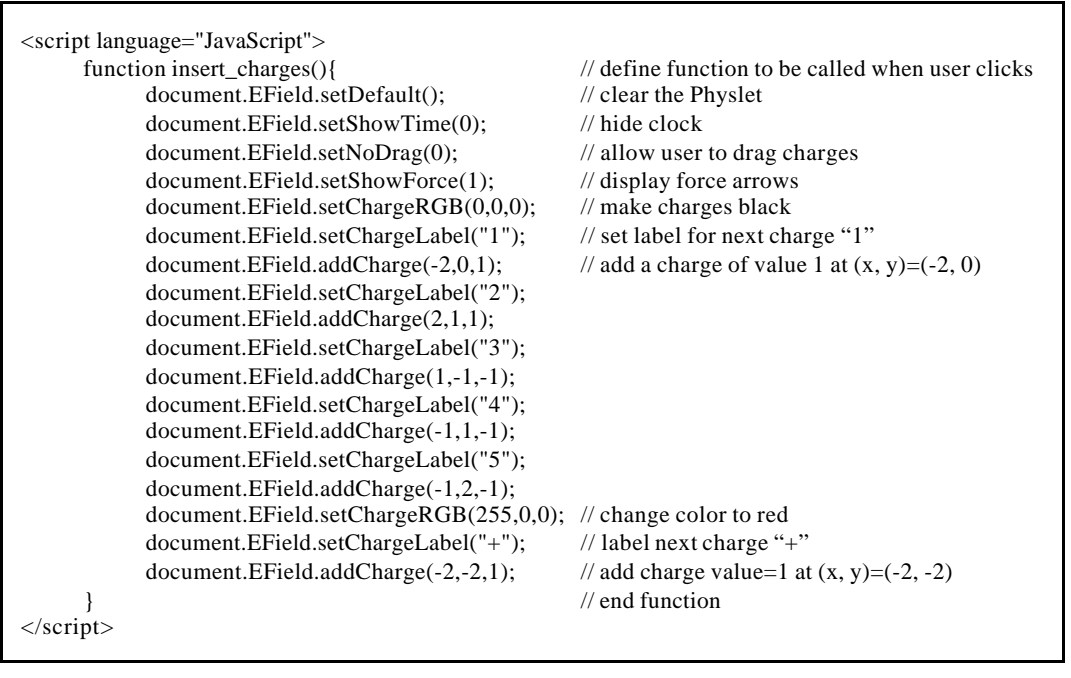


All of these exercises use the same Java applet, EField. JavaScript is used to control the Java applet. This is how different exercises are created. An example script is show in the sidebar. They are available as part of the WebAssign problem database and as html documents. WebAssign is a complete web-based homework system developed at NCSU that provides the instructor the ability to create and assign work from its large library of exercises. It serves students their assignments upon request, collects answers and automatically grades them, and stores the scores for the instructor to access at a later point. For more information about WebAssign, browse http://webassign.net/ or call (800) 955-8275. Alternatively, straight html documents containing these exercises and EField are contained in a zipped file, which may be downloaded from http://webphysics.davidson.edu/Applets/Applets.html and used by instructors in their own web sites. These do not contain any grading mechanisms, but those proficient with cgi programming may add grading capability. The EField Physlet and other Physlets may be obtained from http://webphysics.davidson.edu under the conditions stipulated in that document. That site contains more examples and information on scripting Physlets. Other examples may be found on the website accompanying the Giancoli textbook; go to chapter 16 of http://www.prenhall.com/giancoli/. EField is written in Java 1.1 and therefore requires a recent browser to work. On Windows or UNIX machines, use at a minimum Netscape 4.06 or Internet Explorer 4.01. At the time of this writing, we know of no browsers for the Macintosh that support the JavaScript to communicate with Java 1.1. Although the Physlets themselves run on a Macintosh, they can not be scripted and so these exercises will not work.

Information on finding these exercises in WebAssign:

\begin{tabular}{|l|l|}
\hline Activity & question code \\
\hline Determine the sign of the charge & EField1.3a \\
\hline Coulomb Force & EField1.2 \\
\hline Unknown charge & EField1.1b \\
\hline Electric field representations & EField4.1 \\
\hline Electric flux through a cube & EField3.1 \\
\hline Particle in an uniform electric field & EField4.2 \\
\hline
\end{tabular}

The work described in this paper is part of several projects supported by the National Science Foundation through grants DGE-0071-4007-6802, DUE-9752313, and DUE9752365, by the Department of Education through grant P116B7905, and by the Hewlett-Packard Corporation.

\section{References}

${ }^{1}$ David Trowbridge and Bruce Sherwood, EM Field (Physics Academic Software, Raleigh, NC, 1993, 1996).

${ }^{2}$ Ruth W. Chabay, Electric Field Hockey (Physics Academic Software, Raleigh, NC, 1993,1998).

${ }^{3}$ W. Christian and A. Titus, "Developing Web-based Curricula Using Java Physlets," Computers in Physics 12 (3) 227-232(1998).

${ }^{4}$ WebAssign, WebAssign (North Carolina State University, Raleigh, 1998). http://webassign.net

${ }^{5}$ A. Titus, L. Martin, and R. Beichner, "Web-based Testing in Physics Education: Methods and Opportunities," Computers in Physics 12 (2) 117 123(1998).

${ }^{6}$ Robert Beichner, "SCALE-UP Description" (1998) http://www2.ncsu.edu/ncsu/pams/physics/Physics_Ed /SCALE-UP\%20Description.html

${ }^{7}$ Wolfgang Christian, EField (Davidson College, 1998). http://webphysics.davidson.edu/

${ }^{8}$ A. B. Arons, Teaching Introductory Physics (John Wiley and Sons, New York, 1997) p. 12.

${ }^{9}$ A. B. Arons, Teaching Introductory Physics (John Wiley and Sons, New York, 1997) p. 107. 